\title{
Symbol Based Precoding in The Downlink of Cognitive MISO Channel
}

\author{
Maha Alodeh, Symeon Chatzinotas, and Björn Ottersten \\ Interdisciplinary Centre for Security, Reliability and Trust, University of Luxembourg \\ 4, Alphonse Weicker, 2721 Luxembourg \\ \{maha.alodeh, symeon. chatzinotas, bjorn.ottersten\}@uni.lu
}

\begin{abstract}
This paper proposes symbol level precoding in the downlink of a MISO cognitive system. The new scheme tries to jointly utilize the data and channel information to design a precoding that minimizes the transmit power at a cognitive base station (CBS); without violating the interference temperature constraint imposed by the primary system. In this framework, the data information is handled at symbol level which enables the characterization the intra-user interference among the cognitive users as an additional source of useful energy that should be exploited. A relation between the constructive multiuser transmissions and physical-layer multicast system is established. Extensive simulations are performed to validate the proposed technique and compare it with conventional techniques.
\end{abstract}

\section{Introduction}

The combination of the spectrum scarcity and congestion has motivated researchers to propose more innovative techniques to tackle these challenges. Fixed spectrum allocation techniques assign certain bands to certain applications, which may no longer efficiently used [1. Solving the problem would require changing the regulations which is a complicated and lengthy procedure. With that in mind, the paradigm of cognitive radios has been proposed as a promising agile technology that can revolutionize the future of telecommunication by "breaking the gridlock of the wireless spectrum" 2. The key idea of their implementation is to allow opportunistic transmissions to share the wireless medium. Thus, two initial hierarchical levels have been defined: primary level and cognitive level (the users within each level are called primary users (PU) and cognitive users (CU) respectively). The interaction between these two levels is determined by the agility of the cognitive level and the predefined constraints imposed by the primary level 3 . Overlay, underlay and interweave are three general implementations which regulate the coexistence terms of both systems. The first two

\footnotetext{
* This work is supported by Fond National de la Recherche Luxembourg (FNR) projects, project Smart Resource Allocation for Satellite Cognitive Radio (SRATSCR) ID:4919957 and Spectrum Management and Interference Mitigation in Cognitive Radio Satellite Networks SeMiGod.
} 
implementations allow simultaneous transmissions, which leads to better spectrum utilization in comparison to the last one, which allocates the spectrum to the cognitive system by detecting the absence of the primary one [4.

The form of integration in this work is defined by cooperation between the two levels in the cognitive interference channel. The cooperation can aid the primary network to satisfy the quality of service (QoS) or enhance the rate of its own users by backhauling its data through the cognitive system [5]-8; CBSs can operate as relays for primary messages and as regular base stations to serve their cognitive users. The cognitive system benefits by providing a service to its users. This kind of cognitive implementation fits with practical overlay cognitive definition, as the PU is being served from both the PS and the CBSs by performing relaying between them to make primary data accessible by the CBS. Sometimes the primary symbols are not available to the cognitive system, as a result the cognitive system needs to take the sufficient precautions to protect the primary system from the interference created by its own transmissions. It should be noted that we assume that the CBSs are equipped with multiple antennas to handle multi-user transmissions, and to enable interference mitigation.

The conventional look at interference can be shifted from a degradation factor into a favorable one if we handle the transmitted data frame at symbol level. At this level, the interference can be classified into: constructive and destructive ones. This classification is initially proposed in [12]; instead of fully inverting the channel to grant zero interference among the spatial streams, the proposed precoding suggests keeping the constructive interference while removing the destructive part by partial channel inversion. This technique is proven to outperform the traditional zero forcing precoding. A more advanced technique is proposed in 13, where an interference rotation is examined to make the interference constructive for all users. Moreover, a modified maximum ratio transmissions technique that performs unitary rotations to create constructive interference among the interfering multiuser streams is proposed [15. Furthermore, a connection between symbol based constructive interference precoding and PHY multicast is established in 15- 16 .

In this work, we utilize the symbol level precoding in underlay MISO cognitive radio scenarios. We shape the interference between the cognitive users to provide constructive characteristics without violating the interference temperature constraints on the primary receivers.

\section{System and Signals model}

We consider a cognitive radio network which shares the spectrum resource with a primary network in the underlay mode as fig. (1). The primary network consists of a primary base station (PBS), equipped with $N_{p}$ antennas, serving a single primary user. The cognitive network has a single CBS, equipped with $M$ antennas, serving $K \mathrm{CU}$. Each $\mathrm{CU}$ is equipped with a single antenna. Throughout this paper, we consider that $K \leq M-1$ and that the primary user is equipped 


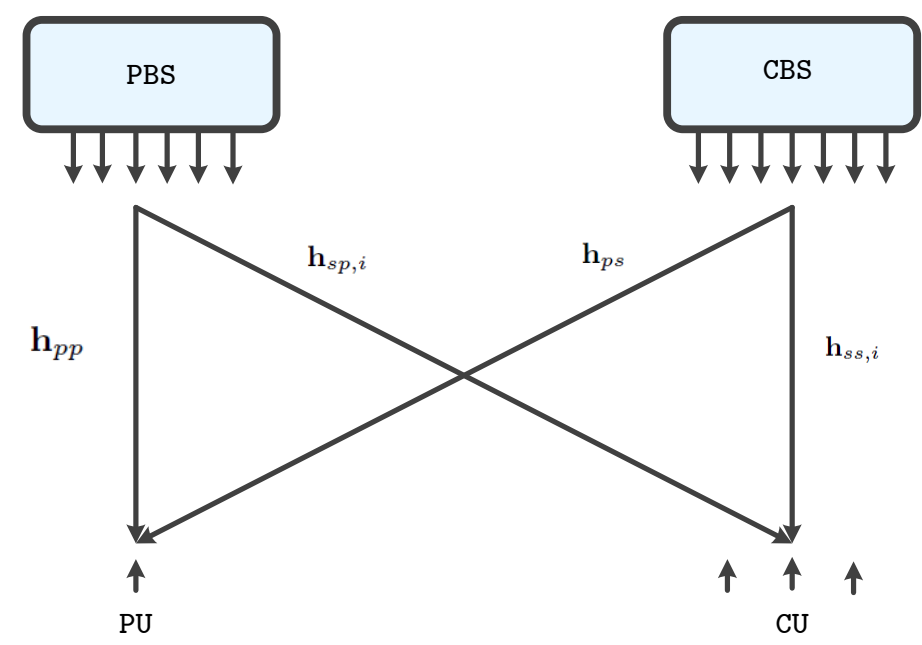

Fig. 1. System model

with a single antenna. Due to the sharing of the same frequency band, the received signal at the primary user is interfered by the signals transmitted from CBS. Similarly, the received signals at the CUs are interfered by the signal transmitted from the PBS.

Assume that in one time slot, a block of information symbols $\mathbf{d}=\left[d_{1}, d_{2}, \ldots, d_{K}\right]^{T}$ are sent from the CBS in which $d_{k}, k=1, \ldots, K$ is the desired signal for user $k$. We assume that $\mathbf{d}$ contains uncorrelated unit-power M-PSK entries. With a proper beamforming (which will be specified later), the transmit signal is given by

$$
\mathbf{x}=\mathbf{W d}
$$

where $\mathbf{W}=\left[\mathbf{w}_{1}, \mathbf{w}_{2}, \ldots, \mathbf{w}_{K}\right]$ denotes the transmit precoding matrix for the cognitive system while $\mathbf{w}_{k} \in \mathbb{C}^{M \times 1}$ denotes the beamforming vector for $k^{\text {th }} \mathrm{CU}$. The received signal at the $k^{t h}$ user, denoted by $y_{s, k}$, is given by

$$
y_{s, k}=\mathbf{h}_{s s, k} \mathbf{w}_{k} d_{k}+\sum_{j \in K, j \neq k} \mathbf{h}_{s s, k} \mathbf{w}_{j} d_{j}+\mathbf{h}_{s p, k} \mathbf{g}^{p} d_{p}+n_{k},
$$

and the received signal at PU's receiver is given by

$$
y_{p}=\mathbf{h}_{p p} \mathbf{g} d_{p}+\sum_{j \in K} \mathbf{h}_{p s} \mathbf{w}_{j} d_{j}+n
$$

where $\mathbf{h}_{s s, k} \in \mathbb{C}^{1 \times M}$ and $\mathbf{h}_{s p, k} \in \mathbb{C}^{1 \times N_{p}}$ are the channels between the CBS and the PBS respectively and the $k^{t h} \mathrm{CU}$. While $\mathbf{h}_{p p}$ and $\mathbf{h}_{p s}$ denote the channel 
between the PBS and PU, CBS and PU respectively. The transmitted power of the primary user is denoted by $p_{p}, \mathbf{g} \in \mathbb{C}^{N_{p} \times 1}$ represents the precoding vector used by the PBS, and $d_{p}$ represents the transmitted symbol from the PBS and it is not available at CBS. Finally, $n_{k} \sim \mathcal{C N}\left(0, \sigma^{2}\right)$ and $n \sim \mathcal{C N}\left(0, \sigma^{2}\right)$ are additive i.i.d. complex Gaussian noise with zero mean and variance $\sigma_{k}^{2}$ at the $k^{\text {th }} \mathrm{CU}$ and PU respectively. The channel state information (CSI) $\mathbf{h}_{p s}$ and $\mathbf{h}_{s s, j}$ are available at the CBS.

\section{Constructive interference Definition}

The interference is a random deviation which can move the desired constellation point in any direction. To address this problem, the power of the interference has been used in the past to regulate its effect on the desired signal point. The interference among the multiuser spatial streams leads to deviation of the received symbols outside of their detection region. However, in symbol level precoding (e.g. M-PSK) this interference pushes the received symbols further into the correct detection region and, as a consequence it enhances the system performance. Therefore, the interference can be classified into constructive or destructive based on whether it facilitates or deteriorates the correct detection of the received symbol. For BPSK and QPSK scenarios, a detailed classification of interference is discussed thoroughly in [12. In this section, we describe the required conditions to have constructive interference for any M-PSK modulation.

\subsection{Constructive Interference Definition}

Assuming both the data symbols and CSI are available at the CBS, the unitpower created interference from the $k^{t h}$ data stream on $j^{\text {th }}$ user can be formulated as:

$$
\rho_{j k}=\frac{\mathbf{h}_{s s, j} \mathbf{w}_{k}}{\left\|\mathbf{h}_{s s, j}\right\|\left\|\mathbf{w}_{k}\right\|} .
$$

Since the adopted modulations are M-PSK ones, a definition for constructive interference can be stated as

Lemma 1 [16] For any M-PSK modulated symbol $d_{k}$, it is said to receive constructive interference from another simultaneously transmitted symbol $d_{j}$ which is associated with $\mathbf{w}_{j}$ if and only if the following inequalities hold

$$
\begin{gathered}
\angle d_{j}-\frac{\pi}{M} \leq \arctan \left(\frac{\mathcal{I}\left\{\rho_{j k} d_{k}\right\}}{\mathcal{R}\left\{\rho_{j k} d_{k}\right\}}\right) \leq \angle d_{j}+\frac{\pi}{M}, \\
\mathcal{R}\left\{d_{k}\right\} \cdot \mathcal{R}\left\{\rho_{j k} d_{j}\right\}>0, \mathcal{I}\left\{d_{k}\right\} \cdot \mathcal{I}\left\{\rho_{j k} d_{j}\right\}>0 .
\end{gathered}
$$

Corollary 1 [16] The constructive interference is mutual. If the symbol $d_{j}$ constructively interferes with $d_{k}$, then the interference from transmitting the symbol $d_{k}$ is constructive to $d_{j}$. 


\section{Constructive interference exploitation}

\subsection{Relaxed Interference Constraint}

The precoding aims at exploiting the constructive interference among the cognitive users without violating the interference temperature constraint imposed by the primary system $\mathcal{I}_{t h}$. The optimization can be formulated as

$$
\begin{aligned}
& \mathbf{w}_{1}, \ldots, \mathbf{w}_{K}=\underset{\mathbf{w}_{1}, \ldots, \mathbf{w}_{K}}{\arg \min }\left\|\sum_{k=1}^{K} \mathbf{w}_{k} d_{k}\right\|^{2} \\
& \text { s.t. } \mathcal{C}_{1}: \angle\left(\mathbf{h}_{s s, j} \sum_{k=1}^{K} \mathbf{w}_{k} d_{k}\right)=\angle\left(d_{j}\right), \forall j \in K \\
& \mathcal{C}_{2}: \frac{\left\|\mathbf{h}_{s s, j} \sum_{k=1}^{K} \mathbf{w}_{k} d_{k}\right\|^{2}}{\sigma^{2}+\left\|\mathbf{h}_{s p, j} \mathbf{g}\right\|^{2}} \geq \zeta_{j} \quad, \forall j \in K \\
& \mathcal{C}_{3}:\left\|\mathbf{h}_{p s} \sum_{k=1} \mathbf{w}_{k} d_{k}\right\|^{2} \leq \mathcal{I}_{t h}
\end{aligned}
$$

The first two sets of constraints $\mathcal{C}_{1}$ and $\mathcal{C}_{2}$ grant the reception of the data symbols with certain SNR level $\zeta_{j}$. The third constraint $\mathcal{C}_{3}$ is to protect the PU from the cognitive systems transmissions. In order to solve (7), we formulate it by using $\mathbf{x}=\sum_{k=1}^{K} \mathbf{w}_{k} d_{k}$ as the following

$$
\begin{array}{lll}
\mathbf{x}=\arg \min _{\mathbf{x}} & \|\mathbf{x}\|^{2} \\
\text { s.t. } & \mathcal{C}_{1}: & \frac{\mathbf{h}_{s s, j} \mathbf{x}+\mathbf{x}^{H} \mathbf{h}_{s s, j}^{H}}{2}=\sqrt{\psi_{i} \zeta_{j}} \mathcal{R}\left\{d_{j}\right\} \quad, \forall j \in K \\
& \mathcal{C}_{2}: & \frac{\mathbf{h}_{s s, j} \mathbf{x}-\mathbf{x}^{H} \mathbf{h}_{s s, j}^{H}}{2 i}=\sqrt{\psi_{i} \zeta_{j}} \mathcal{I}\left\{d_{j}\right\} \quad, \forall j \in K \\
& \mathcal{C}_{3}: & \left\|\mathbf{h}_{p s} \mathbf{x}\right\|^{2} \leq \mathcal{I}_{t h} .
\end{array}
$$

where $\psi_{j}=\sigma^{2}+\left\|\mathbf{h}_{s p, j} \mathbf{g}\right\|^{2}$. To solve the problem, the corresponding Lagrange function can be expressed as

$$
\begin{aligned}
\mathcal{L}(\mathbf{x}) & =\|\mathbf{x}\|^{2} \\
& +\sum_{j} \mu_{j}\left(-0.5 i\left(\mathbf{h}_{s s, j} \mathbf{x}-\mathbf{x}^{H} \mathbf{h}_{s s, j}^{H}\right)-\sqrt{\psi_{j} \zeta} \mathcal{I}\left\{d_{j}\right\}\right) \\
& +\sum_{j} \alpha_{j}\left(0.5\left(\mathbf{h}_{s s, j} \mathbf{x}+\mathbf{x}^{H} \mathbf{h}_{s s, j}^{H}\right)-\sqrt{\psi_{j} \zeta_{j}} \mathcal{R}\left\{d_{j}\right\}\right) \\
& +\lambda\left(\mathbf{x}^{H} \mathbf{h}_{p s}^{H} \mathbf{h}_{p s} \mathbf{x}-\mathcal{I}_{t h}\right) .
\end{aligned}
$$

The KKT conditions can be derived as 


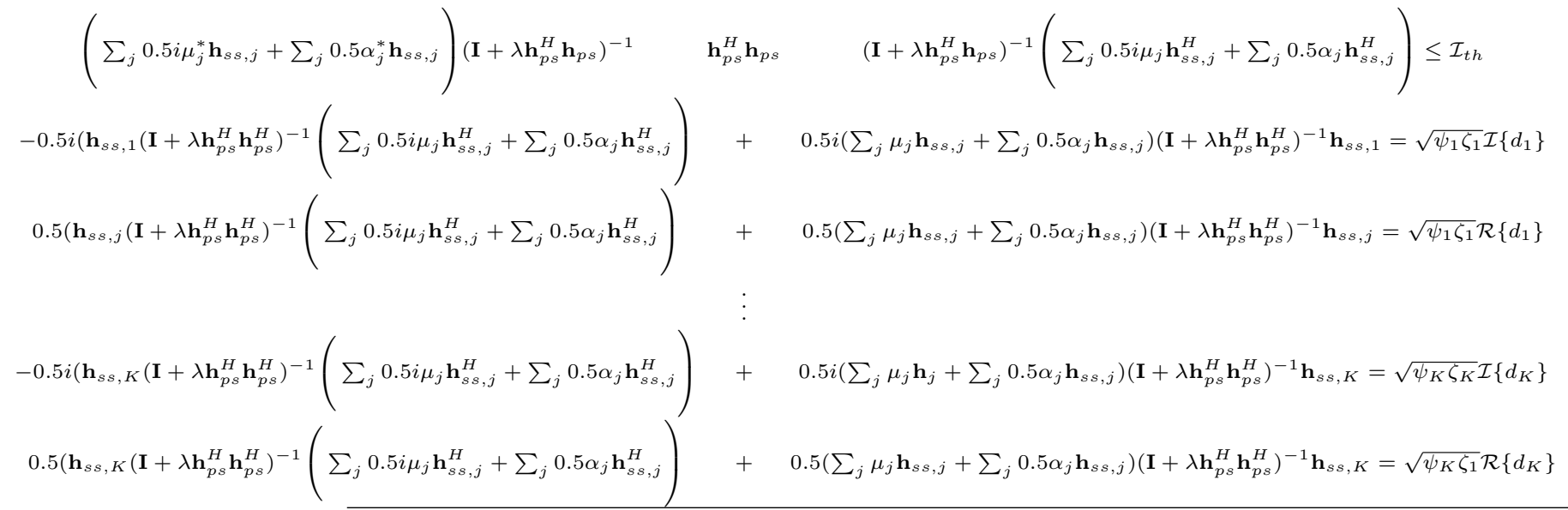

$$
\begin{aligned}
& \frac{d \mathcal{L}\left(\mathbf{x}, \mu_{j}, \alpha_{j}, \lambda\right)}{d \mathbf{x}^{*}}=\mathbf{x}+\sum 0.5 i \mu_{j} \mathbf{h}_{s s, j}^{H}+\sum_{j} 0.5 \alpha_{j} \mathbf{h}_{s s, j}^{H}+\lambda \mathbf{h}_{p s}^{H} \mathbf{h}_{p s} \mathbf{x} \\
& \frac{d \mathcal{L}\left(\mathbf{x}, \mu_{j}, \alpha_{j}, \lambda\right)}{d \mu_{j}}=-0.5 i\left(\mathbf{h}_{s s, j} \mathbf{x}-\mathbf{x}^{H} \mathbf{h}_{s s, j}^{H}\right)-\sqrt{\psi_{j} \zeta_{j}} \mathcal{I}\left\{d_{j}\right\}, \forall j \in K \\
& \frac{d \mathcal{L}\left(\mathbf{x}, \mu_{j}, \alpha_{j}, \lambda\right)}{d \alpha_{j}}=0.5\left(\mathbf{h}_{s s, j} \mathbf{x}+\mathbf{x}^{H} \mathbf{h}_{s s, j}^{H}\right)-\sqrt{\psi_{j} \zeta_{j}} \mathcal{R}\left\{d_{j}\right\}, \forall j \in K \\
& \frac{d \mathcal{L}\left(\mathbf{x}, \mu_{j}, \alpha_{j}, \lambda\right)}{d \lambda}=\left(\mathbf{x}^{H} \mathbf{h}_{p s}^{H} \mathbf{h}_{p s} \mathbf{x}-\mathcal{I}_{t h}\right)
\end{aligned}
$$

By equating $\frac{d \mathcal{L}\left(\mathbf{x}, \mu_{j}, \alpha_{j}, \lambda\right)}{d \mathbf{x}^{*}}$ to zero, we can formulate $\mathbf{x}$ as the following expression

$$
\mathbf{x}=\left(\mathbf{I}+\lambda \mathbf{h}_{p s}^{H} \mathbf{h}_{p s}\right)^{-1}\left(\sum_{j} 0.5 i \mu_{j} \mathbf{h}_{s s, j}^{H}+\sum_{j} 0.5 \alpha_{j} \mathbf{h}_{s s, j}^{H}\right)
$$

By substituting (11) in the set of $(10)$ to form the set of equations 12 , we can find the solution of $\lambda, \alpha_{j}$ and $\mu_{j}$ that satisfies the constraints.

\subsection{Zero Interference Constraint}

If the PU cannot handle any interference, the cognitive transmissions should be in the null space of the channel between CBS and PU. The null space can be defined as

$$
\Pi_{\perp \mathbf{h}_{p s}}=\mathbf{I}-\frac{\mathbf{h}_{p s}^{H} \mathbf{h}_{p s}}{\left\|\mathbf{h}_{p s}\right\|^{2}} .
$$

We design the output vector $\mathbf{x}$ to span the null space of $\mathbf{h}_{p s}$ as the following

$$
\mathbf{x}=\boldsymbol{\Pi}_{\perp \mathbf{h}_{p s}} \hat{\mathbf{x}} .
$$




$$
\begin{aligned}
\arg \min \mathbf{w}_{1}, \mathbf{w}_{2}, \ldots, \mathbf{w}_{K} \quad\left\|\sum_{k=1}^{K} \mathbf{w}_{k} d_{k}\right\|^{2} \\
\text { s.t. } \mathcal{C} 1: \angle\left(\mathbf{h}_{j} \sum_{k=1}^{K} \mathbf{w}_{k} d_{k}\right)=\angle\left(d_{j}\right), \forall j \in K \\
\mathcal{C} 2:\left\|\mathbf{h}_{j} \sum_{k=1}^{K} \mathbf{w}_{k} d_{k}\right\|^{2} \geq \sigma^{2} \zeta_{j} \quad, \forall j \in K \\
\mathcal{C}_{3}:\left\|\mathbf{h}_{p s} \sum_{k=1} \mathbf{w}_{k} d_{k}\right\|^{2}=0 .
\end{aligned}
$$

The previous optimization can be written as The Lagrange function of this optimization problem

$$
\begin{aligned}
\mathcal{L}(\hat{\mathbf{x}}) & =\|\hat{\mathbf{x}}\|^{2} \\
& +\sum_{j} \hat{\mu}_{j}\left(-0.5 i\left(\mathbf{h}_{s s, j} \hat{\mathbf{x}}-\hat{\mathbf{x}}^{H} \mathbf{h}_{s s, j}^{H}\right)-\sqrt{\psi_{j} \zeta_{j}} \mathcal{I}\left\{d_{j}\right\}\right) \\
& +\sum_{j} \hat{\alpha}_{j}\left(0.5\left(\mathbf{h}_{s s, j} \hat{\mathbf{x}}+\hat{\mathbf{x}}^{H} \mathbf{h}_{s s, j}^{H}\right)-\sqrt{\psi_{j} \zeta_{j}} \mathcal{R}\left\{d_{j}\right\}\right) .
\end{aligned}
$$

The KKT condition can be written as

$$
\begin{aligned}
& \frac{d \mathcal{L}\left(\mathbf{x}, \mu_{j}, \alpha_{j}, \lambda\right)}{d \hat{\mathbf{x}}^{*}}=\hat{\mathbf{x}}+\sum 0.5 i \mu_{j} \mathbf{h}_{s s, j}^{H}+\sum_{j} 0.5 \alpha_{j} \mathbf{h}_{s s, j}^{H}+\lambda \mathbf{h}_{p s}^{H} \mathbf{h}_{p s} \hat{\mathbf{x}} \\
& \frac{d \mathcal{L}\left(\mathbf{x}, \mu_{j}, \alpha_{j}, \lambda\right)}{d \mu_{j}}=-0.5 i\left(\mathbf{h}_{s s, j} \hat{\mathbf{x}}-\hat{\mathbf{x}}^{H} \mathbf{h}_{s s, j}^{H}\right)-\sqrt{\psi_{j} \zeta_{j}} \mathcal{I}\left\{d_{j}\right\}, \forall j \in K \\
& \frac{d \mathcal{L}\left(\mathbf{x}, \mu_{j}, \alpha_{j}, \lambda\right)}{d \alpha_{j}}=0.5\left(\mathbf{h}_{s s, j} \hat{\mathbf{x}}+\hat{\mathbf{x}}^{H} \mathbf{h}_{s s, j}^{H}\right)-\sqrt{\psi_{j} \zeta_{j}} \mathcal{R}\left\{d_{j}\right\}, \forall j \in K
\end{aligned}
$$

The solution for the previous optimization problem can be written as

$$
\hat{\mathbf{x}}=\sum_{j} 0.5 i \hat{\mu}_{j} \mathbf{h}_{s s, j}^{H}+\sum_{j} 0.5 \hat{\alpha}_{j} \mathbf{h}_{s s, j}^{H}
$$

where $\hat{\mu}_{j}, \hat{\alpha}_{j}$ can be found by solving the set of equation (19). Hence, the final formulation for the solution at zero interference temperature constraint

$$
\mathbf{x}=\left(\mathbf{I}-\frac{\mathbf{h}_{p s}^{H} \mathbf{h}_{p s}}{\left\|\mathbf{h}_{p s}\right\|^{2}}\right)\left(\sum_{j} 0.5 i \hat{\mu}_{j} \mathbf{h}_{s s, j}^{H}+\sum_{j} 0.5 \hat{\alpha}_{j} \mathbf{h}_{s s, j}^{H}\right)
$$

\section{Theoretical upper-bound}

The theoretical upper-bound can be formulated by dropping the phase constraint $\mathcal{C}_{1}$ of 77 . The optimal input covariance $\mathbf{Q}$ can be found by solving the following optimization problem: 


$$
\begin{aligned}
& 0.5 K\left\|\mathbf{h}_{s 1}\right\|\left(\sum_{k}\left(-\mu_{k}+\alpha_{k} i\right)\left\|\mathbf{h}_{s s, k}\right\| \rho_{1 k}\right.\left.-\sum_{k}\left(-\mu_{k}+\alpha_{k} i\right)\left\|\mathbf{h}_{s s, k}\right\| \rho_{1 k}^{*}\right)=\sqrt{\psi_{1} \zeta_{1}} \mathcal{I}\left(d_{1}\right) \\
& 0.5 K\left\|\mathbf{h}_{s s, 1}\right\|\left(\sum_{k}\left(-\mu_{k} i-\alpha_{k}\right)\left\|\mathbf{h}_{s s, k}\right\| \rho_{1 k}\right.\left.+\sum_{k}\left(-\mu_{k} i-\alpha_{k}\right)\left\|\mathbf{h}_{s s, k}\right\| \rho_{1 k}^{*}\right)=\sqrt{\psi_{1} \zeta_{1}} \mathcal{R}\left(d_{1}\right) \\
& \vdots \\
& 0.5 K\left\|\mathbf{h}_{s s, K}\right\|\left(\sum_{k}\left(-\mu_{k}+\alpha_{k} i\right)\left\|\mathbf{h}_{s s, k}\right\| \rho_{K k}-\sum_{k}\left(-\mu_{k}+\alpha_{k} i\right)\left\|\mathbf{h}_{s s, k}\right\| \rho_{K k}^{*}\right)=\sqrt{\psi_{K} \zeta_{K}} \mathcal{I}\left(d_{K}\right) \\
& 0.5 K\left\|\mathbf{h}_{s, K}\right\|\left(\sum_{k}\left(-\mu_{k} i-\alpha_{k}\right)\left\|\mathbf{h}_{s s, k}\right\| \rho_{K k}+\sum_{k}\left(-\mu_{k} i-\alpha_{k}\right)\left\|\mathbf{h}_{s s, K}\right\| \rho_{K k}^{*}\right)=\sqrt{\psi_{K} \zeta_{K}} \mathcal{R}\left(d_{K}\right)
\end{aligned}
$$

$$
\begin{aligned}
\mathbf{Q}= & \arg \min _{\mathbf{Q}} \operatorname{tr}(\mathbf{Q}) \\
\text { s.t. } & \mathbf{h}_{s s, j} \mathbf{Q} \mathbf{h}_{s s, j}^{H}=\psi_{j} \gamma_{j} \forall j \in K . \\
& \mathbf{h}_{p s} \mathbf{Q} \mathbf{h}_{p s}^{H} \leq \mathcal{I}_{t h} .
\end{aligned}
$$

where $\mathbf{Q}=\mathbf{x x}^{H}$. This problem resembles the multicast problem [10] with additional interference temperature constraint to suit the constraint imposed by the primary system.

\section{Numerical results}

In order to assess the performance of the proposed transmissions schemes, MonteCarlo simulations of the different algorithms have been conducted to study the performance of the proposed techniques and compare to the state of the art techniques. The adopted channel model is assumed to be as the following

- $\mathbf{h}_{p p} \sim \mathcal{C N}\left(0, \sigma_{p p}^{2} \mathbf{1}_{1 \times M}\right)$, where $\mathbf{1}_{1 \times M}$ is vector of all ones and of size $1 \times M$.

$-\mathbf{h}_{p s} \sim \mathcal{C N}\left(0, \sigma_{p s}^{2} \mathbf{1}_{1 \times M}\right)$

- $\mathbf{h}_{s s, j} \sim \mathcal{C N}\left(0, \sigma_{s s}^{2} \mathbf{1}_{1 \times M}\right), \forall j \in K$

$-\mathbf{h}_{s p, j} \sim \mathcal{C N}\left(0, \sigma_{s p, j}^{2} \mathbf{1}_{1 \times M}\right), \forall j \in K$

- To study the performance of the system at the worst case scenario, when all users have a strong channel with respect to its direct and interfering base stations $\sigma_{p p}^{2}=\sigma_{p s}^{2}=\sigma_{s p, j}^{2}=\sigma_{s s}^{2}=\sigma^{2}$.

In the figures, we denote the proposed cognitive technique that exploits the constructive interference by (CCIPM), while $\mathrm{S}$ denotes the strict interference constraints $\mathcal{I}_{t h}=0$. CBS has 3 antennas and serves 2 cognitive users. We compare the performance of the proposed scheme (CCIPM) to the scheme in [13] tailored to cognitive scenario by solving the following optimization

$$
\begin{array}{rl}
\mathbf{W}_{C C I Z F}=\min _{\mathbf{W}} & \mathbb{E}\left\{\left\|\mathbf{R}_{\phi}-\mathbf{H}_{s s} \mathbf{W} \mathbf{d}\right\|^{2}\right\} \\
\text { s.t. } & \|\mathbf{W}\|^{2} \leq P \\
& \mathbf{h}_{p s} \mathbf{W} \mathbf{W}^{H} \mathbf{h}_{p s}^{H}=0
\end{array}
$$

where $\mathbf{R}_{\phi}$ is defined in [13]. We utilize the energy efficiency metric to assess the performance of the proposed technique as 


$$
\eta=\frac{\sum_{j=1}^{K} R_{j}}{\|\mathbf{x}\|^{2}},
$$

$R_{j}$ denotes the rate of the $j^{\text {th }}$ user. In all figures, we depict the performance of constructive interference zero forcing (CCIZF). For the sake of comparison, the transmit power of the CCIZF solutions can be scaled until all users achieve the target rate.

In Fig 2, the energy efficiency with respect to the average channel is depicted, the used modulation is QPSK and the strict interference constraint imposed by the primary system. We compare the performance of the CCIPM to the theoretical upper-bound and CCIZF. It can be noted that the CCIZF curve saturates at the low-mid SNR regime, while the curves of CCIPM and multicast have higher growth in terms of energy efficiency in the same regime. Moreover, it can be noted that CCIPM outperforms CCIZF at different channel strength values.

In Fig. 3, we depict the energy efficiency at different target rates. For the constructive interference schemes, we assign the target rate with its corresponding MPSK modulation. It can be noted that the theoretical upper bounds for the scenario of the strict and the relaxed interference constraints have the same power consumption at target rate equals to $1 \mathrm{bps} / \mathrm{Hz}$. However, this result does not hold for constructive interference technique. Moreover, it can be noted that the gap between the theoretical bound and the CCIPM is fixed at the both scenario for all target rates.

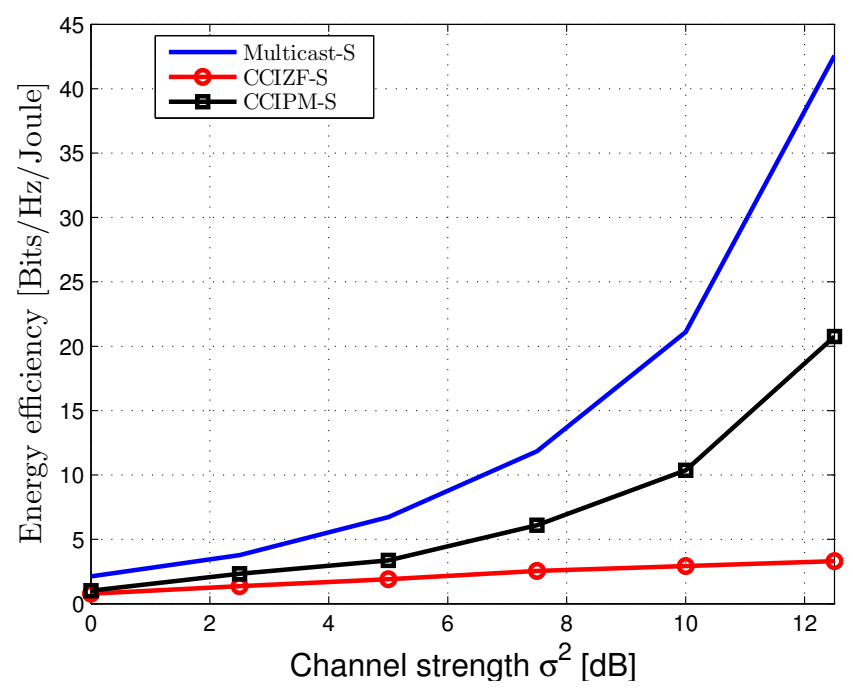

Fig. 2. Energy efficiency vs channel strength. The adopted modulation is QPSK for $C C I Z F$ and $C C I P M, \zeta=4.7712 d B$. 


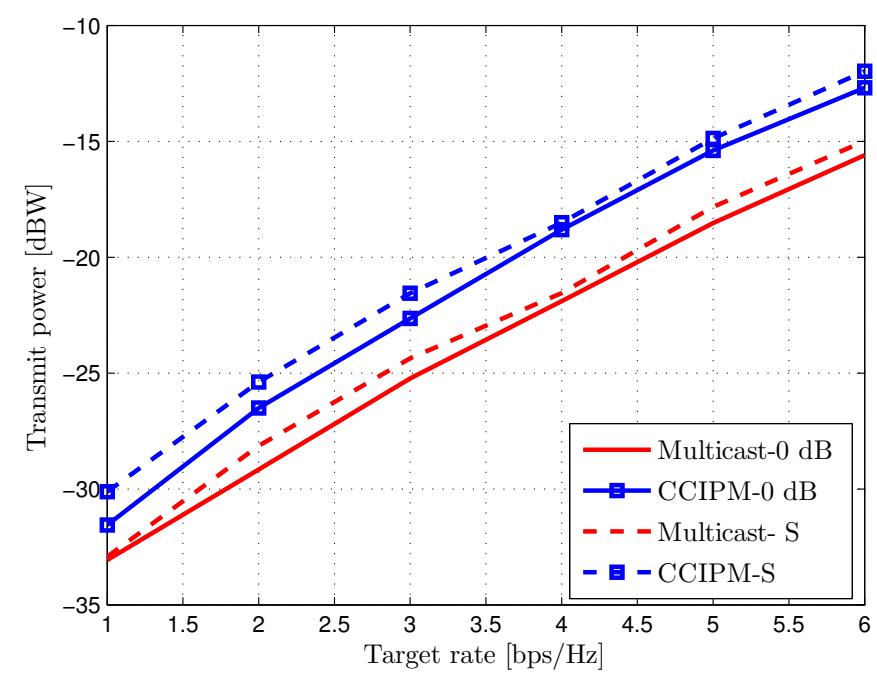

Fig. 3. Transmit power vs target rate. $\sigma^{2}=10 d B$

\section{Conclusions}

In this paper, we propose symbol-level precoding techniques for the downlink of cognitive underlay system. These techniques exploits the availability of the CSI and the data symbols to constructively correlate the transmission for the cognitive users without violating the interference temperature at the primary users. This enables interference exploitation among the cognitive multiuser transmissions assuming M-PSK modulation. The designed precoder aims at minimizing the transmitted power at CBS while granting a certain received SNR at each cognitive users. From the numerical results section, it can be concluded that the CBS consumes less power at the relaxed interference constraints. Finally, a comparison with the theoretical upperbound and the state-of-the art techniques is illustrated.

\section{References}

1. Federal Communication Commission, Spectrum Policy Task Force, ET document no. 02-135, Nov. 2002.

2. A. Goldsmith, S. A. Jafar, I. Maric, and S. Srinivasa, "Breaking spectrum gridlock with cognitive radios: An information theoretic perspective," IEEE, vol. 97, no. 5, pp. 894 - 914, May 2009.

3. S. Haykin, "Cognitive Radio: Brain-Empowered Wireless Communications," IEEE Journal on Selected Areas in Communications, vol. 23, pp. 201-22, Feb. 2005.

4. S. Srinivasa, S. A. Jafar, "Soft Sensing and Optimal Power Control for Cognitive Radio," IEEE Transactions on Wireless Communications, vol. 9, no. 12, pp. 36383649, October 2010. 
5. J. Lv, R. Blasco-Serrano, E. Jorswieck, R. Thobaben and A. Kliks, "Optimal Beamforming in MISO Cognitive Channels with Degraded Message Sets," IEEE Conference on Wireless Communications and Networking (WCNC), April 2012.

6. J. Lv, R. Blasco-Serrano, E. Jorswieck and R. Thobaben, "Linear Precoding in MISO Cognitive Channels with Causal Primary Message," IEEE International Symposium on Wireless Communications Systems (ISWCS), 2012.

7. J. Lv, R. Blasco-Serrano, E. Jorswieck and R. Thobaben, "Multi-antenna transmission for underlay and overlay cognitive radio with explicit message-learning phase,"EURASIP Journal on Wireless Communications and Networking (JWCN), special issue on "Cooperative Cognitive Networks", Jul. 2013.

8. G. Zheng, S. H. Song, K. K. Wong, and B. Ottersten, "Cooperative Cognitive Networks: Optimal, Distributed and Low-Complexity Algorithms," IEEE Transaction on Signal Processing, vol. 61, no.11, pp. 2778 - 2790, June 2013.

9. S. H. Song and K.B. Letaief, "Prior Zero-Forcing for Relaying Primary Signals in Cognitive Network," IEEE Global Telecommunication Conference (GLOBECOM), December 2010.

10. N. D. Sidropoulos, T. N. Davidson, ans Z.-Q. Luo, "Transmit Beamforming for Physical-Layer Multicasting," IEEE Transactions on Signal Processing, vol. 54, no. 6, pp. 2239-2251, June 2006.

11. N. Jindal and Z.-Q. Luo, "Capacity Limits of Multiple Antenna Multicast," IEEE International Symposium on Information Theory (ISIT), pp. 1841 - 1845, June 2006.

12. C. Masouros and E. Alsusa, "Dynamic Linear Precoding for the exploitation of Known Interference in MIMO Broadcast Systems," IEEE Transactions On Communications, vol. 8, no. 3, pp. 1396 - 1404, March 2009.

13. C. Masouros, "Correlation Rotation Linear Precoding for MIMO Broadcast Communications," IEEE Transaction on Signal Processing, vol. 59, no. 1, pp. 252 -262, January 2011.

14. M. Alodeh, S. Chatzinotas and B. Ottersten, "Data Aware User Selection in the Cognitive Downlink MISO Precoding Systems," invited paper to IEEE International Symposium on Signal Processing and Information Technology (ISSPIT), December 2013.

15. M. Alodeh, S. Chatzinotas and B. Ottersten, "A Multicast Approach for Constructive Interference Precoding in MISO Downlink Channel," in the proceedings of International Symposium in Information theory (ISIT) 2014, Available on arXiv:1401.6580v2 [cs.IT].

16. M. Alodeh, S. Chatzinotas and B. Ottersten, "Constructive Multiuser Interference in Symbol Level Precoding for the MISO Downlink Channel," IEEE Transactions on Signal processing 2015, Available on arXiv:1408.4700 [cs.IT]. 